PROCEEDINGS OF THE

AMERICAN MATHEMATICAL SOCIETY

Volume 134, Number 1, Pages 55-65

S 0002-9939(05)08176-1

Article electronically published on August 15, 2005

\title{
BUCHSBAUM STANLEY-REISNER RINGS WITH MINIMAL MULTIPLICITY
}

\author{
NAOKI TERAI AND KEN-ICHI YOSHIDA
}

(Communicated by Bernd Ulrich)

\begin{abstract}
In this paper, we study Buchsbaum Stanley-Reisner rings with linear free resolution. We introduce the notion of Buchsbaum Stanley-Reisner rings with minimal multiplicity of initial degree $q$, which extends the notion of Buchsbaum rings with minimal multiplicity defined by Goto. As an application, we give many examples of non-Cohen-Macaulay Buchsbaum StanleyReisner rings with linear resolution.
\end{abstract}

\section{INTRODUCTION}

For any simplicial complex $\Delta$ on $V=\left\{x_{1}, \ldots, x_{n}\right\}$, the homogeneous reduced $k$-algebra $k[\Delta]=k\left[X_{1}, \ldots, X_{n}\right] / I_{\Delta}$, where $I_{\Delta}$ is the ideal generated by all squarefree monomials $X_{i_{1}} \cdots X_{i_{p}}$ such that $\left\{x_{i_{1}}, \ldots, x_{i_{p}}\right\} \notin \Delta$, is called the StanleyReisner ring of $\Delta$. In recent years, the class of Stanley-Reisner rings is one of important classes in the theory of commutative algebra. A homogeneous $k$-algebra $A$ is said to have q-linear resolution if its graded minimal free resolution of $A$ over $S=k\left[X_{1}, \ldots, X_{n}\right]$ has the following shape:

$$
0 \rightarrow S(-(q+p-1))^{\beta_{p}} \rightarrow S(-(q+p-2))^{\beta_{p-1}} \rightarrow \cdots \rightarrow S(-q)^{\beta_{1}} \rightarrow S \rightarrow A \rightarrow 0 .
$$

In [4, Eisenbud and Goto investigated rings with linear resolution and showed the significance of this property. Then from many viewpoints, the rings with linear resolution have been widely studied. Let us pick up some important results in the class of Stanley-Reisner rings. Fröberg [5, 6] classified all $\Delta$ for which $k[\Delta]$ has 2-linear resolution. Hibi [10] gave a necessary and sufficient condition for a Buchsbaum Stanley-Reisner ring to have linear resolution in terms of the reduced homology of the simplicial complex and the $a$-invariants of its links. Eagon and Reiner [3] proved that $k[\Delta]$ has linear resolution if and only if $k\left[\Delta^{*}\right]$ is CohenMacaulay, where $\Delta^{*}$ denotes the Alexander dual of $\Delta$.

Also, there is a well-known criterion for a Cohen-Macaulay (Stanley-Reisner) ring to have linear resolution in terms of its $h$-vector or its multiplicity with given initial degree and codimension (see e.g. 4]). However, as for the Buchsbaum case, it seems that there is no such criterion. Hence, in this paper, we investigate the

Received by the editors August 28, 2004.

2000 Mathematics Subject Classification. Primary 13F55; Secondary 13D02.

Key words and phrases. Stanley-Reisner ring, Buchsbaum ring, regularity, linear resolution, Alexander duality, minimal multiplicity.

(C)2005 American Mathematical Society Reverts to public domain 28 years from publication 
structure of Buchsbaum Stanley-Reisner rings with linear resolution in connection with their multiplicities.

Let us explain the organization of this paper. In Section 1, we give fundamental properties of Buchsbaum Stanley-Reisner rings with linear resolution. Now suppose that $A=k[\Delta]$ is a $d$-dimensional Buchsbaum ring with $q$-linear resolution $(2 \leq q \leq$ $d$ ), and put $\operatorname{codim} A=c \geq 1$. Then since $H_{\mathfrak{m}}^{i}(A)=0$ for all $i \neq q-1, d$, it seems that $h:=\operatorname{dim}_{k} H_{\mathfrak{m}}^{q-1}(A)$ is an important invariant of $A$. From this point of view, we determine the $h$-vector of $A$ and prove an inequality:

$$
0 \leq h \leq h_{c, d, q}:=\frac{(c+q-2) \cdots(c+1) c}{d(d-1) \cdots(d-q+2)} ;
$$

see Theorem 1.3. Also, we study the Alexander dual of Buchsbaum complexes with linear resolution.

In Section 2, we introduce the notion of minimal multiplicity of initial degree $q$ for Buchsbaum Stanley-Reisner rings, and investigate its property. In [8], Goto defined Buchsbaum local rings with minimal multiplicity, and he proved that they have 2-linear resolutions; see [7, 8]. In fact, we generalize this notion in the class of Stanley-Reisner rings and prove that a $d$-dimensional Buchsbaum ring $A=k[\Delta]$ has minimal multiplicity of initial degree $q$ if and only if it has $q$-linear resolution and $\operatorname{dim}_{k} H_{\mathfrak{m}}^{q-1}(A)=h_{c, d, q}$; see Theorem 2.3 for more details.

In Section 3, we give several examples of Buchsbaum Stanley-Reisner rings with minimal multiplicity. In particular, we show that the Alexander dual of the boundary complex of a cyclic polytope is Buchsbaum with minimal multiplicity in our sense. As an application, we can answer the following problem.

Problem (Hibi). Construct a non-Cohen-Macaulay Buchsbaum complex of dimension $d-1$ with $q$-linear resolution for any given integers $q, d$ with $2 \leq q \leq d$.

\section{Buchsbaum Stanley-Reisner Rings With $q$-Linear Resolution}

We first fix notation. Let $\mathbb{N}$ (resp. $\mathbb{Z}$ ) denote the set of non-negative integers (resp. integers). Let $\#(W)$ denote the cardinality of a set $W$.

We recall some notation on simplicial complexes and Stanley-Reisner rings according to [13]. We refer the reader to e.g. [1, 13, for the detailed information about combinatorial and algebraic background. Let $\Delta$ be a simplicial complex on a vertex set $V=\left\{x_{1}, \ldots, x_{n}\right\}$. Put $S=k\left[X_{1}, \ldots, X_{n}\right]$. Let $k[\Delta]=S / I_{\Delta}$ denote the Stanley-Reisner ring of $\Delta$ over a field $k$. We say that $k[\Delta]$ is Buchsbaum if and only if $H_{\mathfrak{m}}^{i}(k[\Delta])=\left[H_{\mathfrak{m}}^{i}(k[\Delta])\right]_{0}$ for all $i<d=\operatorname{dim} k[\Delta]$. Then we put

$$
I(k[\Delta])=\sum_{i=0}^{d-1}\left(\begin{array}{c}
d-1 \\
i
\end{array}\right) l\left(H_{\mathfrak{m}}^{i}(k[\Delta])\right)=\sum_{i=0}^{d-1}\left(\begin{array}{c}
d-1 \\
i
\end{array}\right) \operatorname{dim}_{k} \widetilde{H}_{i-1}(\Delta ; k),
$$

where $\widetilde{H}_{p}(\Delta ; k)$ denotes the $p$ th reduced simplicial homology group of $\Delta$ with values in $k$.

Let $A=k\left[A_{1}\right]=S / I$ be a homogeneous $k$-algebra with the unique homogeneous maximal ideal $\mathfrak{m}$, and suppose that $I \subseteq \mathfrak{m}^{2}$ unless otherwise specified. A graded minimal free resolution of $A$ over $S$ is an exact sequence

$$
0 \rightarrow \bigoplus_{j \in \mathbb{Z}} S(-j)^{\beta_{p, j}(A)} \stackrel{\varphi_{p}}{\longrightarrow} \cdots \stackrel{\varphi_{2}}{\longrightarrow} \bigoplus_{j \in \mathbb{Z}} S(-j)^{\beta_{1, j}(A)} \stackrel{\varphi_{1}}{\longrightarrow} S \rightarrow A \rightarrow 0,
$$


where $S(j), j \in \mathbb{Z}$, denotes the graded module $S(j)=\bigoplus_{n \in \mathbb{Z}} S_{j+n}$ and "minimal" means that $\varphi_{i} \otimes_{S} S / \mathfrak{m}=0$ for all $i$. We call $\beta_{i}(A)=\sum_{j \in \mathbb{Z}} \beta_{i, j}(A)$ the $i$ th Bett $i$ number of $A$ over $S$. The Castelnuovo-Mumford regularity is defined by

$$
\operatorname{reg} A:=\max \left\{j-i: \beta_{i, j}(A) \neq 0\right\} .
$$

By [4, we can compute the regularity in terms of local cohomology modules:

$$
\operatorname{reg} A=\inf \left\{r \in \mathbb{Z}:\left[H_{\mathfrak{m}}^{i}(A)\right]_{j}=0 \text { for all } j>r-i\right\} .
$$

Also, the initial degree of $A$ is defined by

$$
\operatorname{indeg} A:=\min \left\{j: I_{j} \neq 0\right\}=\min \left\{j: \beta_{1, j}(A) \neq 0\right\} .
$$

Then $\operatorname{reg} A \geq \operatorname{indeg} A-1$. We say that $A$ has $q$-linear resolution (abbr., $A$ is $q$ linear) if $\operatorname{reg} A=\operatorname{indeg} A-1=q-1$, that is, its graded minimal free resolution of $A$ can be written as the following shape:

$$
0 \rightarrow S(-(q+p-1))^{\beta_{p}} \rightarrow S(-(q+p-2))^{\beta_{p-1}} \rightarrow \cdots \rightarrow S(-q)^{\beta_{1}} \rightarrow S \rightarrow A \rightarrow 0 .
$$

We recall the definition of a-invariant of $A$ :

$$
a(A)=\max \left\{m \in \mathbb{Z}:\left[H_{\mathfrak{m}}^{d}(A)\right]_{m} \neq 0\right\} .
$$

Then we always have $a(k[\Delta]) \leq 0$.

In the following, let us gather several properties of Buchsbaum Stanley-Reisner rings having $q$-linear resolution. In the following, we put $d=\operatorname{dim} A, c=\operatorname{codim} A$ and $q=\operatorname{indeg} A$, unless otherwise specified.

Proposition 1.1 (cf. 44). Suppose that $A=k[\Delta]$ is Buchsbaum with q-linear resolution. Then:

(1) $I_{\Delta}$ is generated by some monomials of degree $q$.

(2) $q \leq d+1$.

(3) $H_{\mathfrak{m}}^{i}(A)=\left[H_{\mathfrak{m}}^{i}(A)\right]_{0} \cong \widetilde{H}_{i-1}(\Delta ; k)=0$ for all $i \neq q-1, d$.

(4) $a(A)=q-d-2$ or $q-d-1$.

Also, suppose that $q \leq d$. Then:

(5) $\widetilde{H}_{d-1}(\Delta ; k)=0$.

(6) $\operatorname{dim}_{k} H_{\mathfrak{m}}^{q-1}(A)=\operatorname{dim}_{k} \widetilde{H}_{q-2}(\Delta ; k)=\beta_{c+d-q+1}$.

Proof. (1) is clear by definition. (2) follows from the fact that $\operatorname{reg} A \leq d$ holds in general by Hochster's formula for local cohomology modules (see [1, Theorem 5.3.8] or [13, Theorem 4.1]). Also, (3) easily follows from [4, Corollary 1.15] and the Buchsbaumness of $A=k[\Delta]$. Similarly, we have $a(A) \leq q-d-1$. On the other hand, $q-1=\operatorname{reg}(A) \leq a(A)+d+1$ by [11, Corollary 2.8]. This implies that $a(A) \geq q-d-2$. Hence we get (4).

Now, suppose that $q \leq d$. Since $d>q-1=\operatorname{reg} A$, we have that $\widetilde{H}_{d-1}(\Delta ; k)=$ $\left[H_{\mathfrak{m}}^{d}(A)\right]_{0}=0$. (6) follows from Hochster's formula on the Betti numbers; see [13, Theorem 4.8].

In the following, we always assume that $q \leq d$. In fact, we can completely characterize Stanley-Reisner rings with $(d+1)$-linear resolution as follows:

Proposition 1.2. Let $A=k[\Delta]$ be a d-dimensional Stanley-Reisner ring on $V$. Then the following conditions are equivalent:

(1) A has $(d+1)$-linear resolution.

(2) $\operatorname{indeg} A=d+1$. 
(3) $I_{\Delta}=\left(X_{i_{1}} \cdots X_{i_{d+1}}: 1 \leq i_{1}<\cdots<i_{d+1} \leq n\right)$. That is, $\Delta$ is the $(d-1)$ skeleton of the standard $(n-1)$-simplex $2^{V}$.

When this is the case, $A$ is Cohen-Macaulay.

Proof. It is easy to check (3) $\Longrightarrow(2)$ and (1) $\Longrightarrow(2)$. Also (2) $\Longrightarrow(1)$ follows from $\operatorname{reg} A \leq d$. Thus it is enough to show (2) $\Longrightarrow(3)$. Suppose that indeg $A=$ $d+1$. Then $I_{\Delta}$ does not contain any square-free monomial $M$ with $\operatorname{deg} M \leq d$. Now suppose that some square-free monomial $X_{i_{1}} \cdots X_{i_{d+1}}$ of degree $(d+1)$ is not contained in $I_{\Delta}$. Then $\left\{x_{i_{1}}, \ldots, x_{i_{d+1}}\right\} \in \Delta$. This contradicts the assumption that $\operatorname{dim} \Delta=d-1$. Hence $\Delta$ is a $(d-1)$-skeleton of $2^{V}$. Since $2^{V}$ is Cohen-Macaulay, so is $\Delta$; see e.g., [1, Ex. 5.1.23].

Now suppose that $A=k[\Delta]$ is a $d$-dimensional Buchsbaum Stanley-Reisner ring with $q$-linear resolution. Then we focus on $h:=\operatorname{dim}_{k} \widetilde{H}_{q-2}(\Delta ; k)=\operatorname{dim}_{k} H_{\mathrm{m}}^{q-1}(A)$, which is an important invariant of $\Delta$. Let $e(A)$ denote the multiplicity of $A$. The following theorem plays an important role in this article.

Theorem 1.3. Suppose that $A=k[\Delta]$ is Buchsbaum and $2 \leq q \leq d$. Put $h=$ $\operatorname{dim}_{k} H_{\mathfrak{m}}^{q-1}(A)$. Then the following conditions are equivalent:

(1) A has q-linear resolution.

(2) The h-vector $\left(h_{0}, h_{1}, \ldots, h_{q-1}, h_{q}, h_{q+1}, \ldots, h_{d}\right)$ of $\Delta$ is

$$
\left(1, c, \cdots,\left(\begin{array}{c}
c+q-2 \\
q-1
\end{array}\right),-\left(\begin{array}{l}
d \\
q
\end{array}\right) h,\left(\begin{array}{c}
d \\
q+1
\end{array}\right) h, \cdots,(-1)^{d-q+1}\left(\begin{array}{l}
d \\
d
\end{array}\right) h\right) .
$$

(3) The following equalities hold:

$$
e(A)=\left(\begin{array}{c}
c+q-1 \\
q-1
\end{array}\right)-h\left(\begin{array}{l}
d-1 \\
q-1
\end{array}\right) \quad \text { and } \quad I(A)=h\left(\begin{array}{l}
d-1 \\
q-1
\end{array}\right) .
$$

When this is the case, the following inequalities hold:

$$
0 \leq h \leq \frac{(c+q-2) \cdots(c+1) c}{d(d-1) \cdots(d-q+2)}=: h_{c, d, q} .
$$

Proof. First suppose (1), that is, $A$ is $q$-linear. Since indeg $A=q$, one has

$$
h_{p}=\left(\begin{array}{c}
c+p-1 \\
p
\end{array}\right) \text { for all } p=0,1, \ldots, q-1 .
$$

On the other hand, by the similar argument as in the proof of [15, Theorem 2.1], we have

$$
\begin{aligned}
0 & =\operatorname{dim}_{k}\left[H_{\mathfrak{m}}^{d}(A)\right]_{-1}=d \cdot h_{d}+h_{d-1}, \\
0 & =\operatorname{dim}_{k}\left[H_{\mathfrak{m}}^{d}(A)\right]_{-2}=\left(\begin{array}{c}
d+1 \\
2
\end{array}\right) h_{d}+d \cdot h_{d-1}+h_{d-2}, \\
\ldots \ldots & \\
0 & =\operatorname{dim}_{k}\left[H_{\mathfrak{m}}^{d}(A)\right]_{q-d}=\left(\begin{array}{c}
2 d-q-1 \\
d-q
\end{array}\right) h_{d}+\left(\begin{array}{c}
2 d-q-2 \\
d-q-1
\end{array}\right) h_{d-1}+\cdots+h_{q} .
\end{aligned}
$$

By Proposition [.1, we have

$$
(-1)^{d-1} h_{d}=\widetilde{\chi}(\Delta)=\sum_{i=-1}^{d-1}(-1)^{i} \operatorname{dim}_{k} \widetilde{H}_{i}(\Delta ; k)=(-1)^{q} h .
$$


Solving the above linear equations on $h_{q}, \ldots, h_{d}$, one can easily obtain that $h_{p}=$ $(-1)^{p-q+1}\left(\begin{array}{l}d \\ p\end{array}\right) h$ for all $p=q, \ldots, d-1, d$. Thus we get $(2)$. Then

$$
\left(\begin{array}{c}
2 d-q \\
d-q+1
\end{array}\right) h_{d}+\cdots+d \cdot h_{q}+h_{q-1}=\operatorname{dim}_{k}\left[H_{\mathfrak{m}}^{d}(A)\right]_{q-d-1} \geq 0
$$

implies that

$$
\left(\begin{array}{c}
c+q-2 \\
q-1
\end{array}\right)-\left(\begin{array}{c}
d \\
q-1
\end{array}\right) h \geq 0 .
$$

Namely, $h \leq h_{c, d, q}$, as required. Since it is easy to see $(2) \Longrightarrow(3)$, it suffices to show $(3) \Longrightarrow(1)$. If necessary, we may assume that $k$ is infinite. Let $J$ be a homogeneous minimal reduction of $\mathfrak{m}$, that is, $J$ is a homogeneous parameter ideal of $A$ and $\mathfrak{m}^{r+1}=J \mathfrak{m}^{r}$ holds for some integer $r \geq 0$. Since $A$ is Buchsbaum and $B:=A / J$ is a homogeneous Artinian $k$-algebra with $\operatorname{dim}_{k} B_{1}=c$ and indeg $B \geq q$, we have

$$
e(A)=l_{A}(A / J)-I(A) \geq l_{A}\left(A / J+\mathfrak{m}^{q}\right)-I(A)=\left(\begin{array}{c}
c+q-1 \\
q-1
\end{array}\right)-I(A)=e(A),
$$

where the last equality follows from the assumption (3). Thus $\mathfrak{m}^{q}=J \cap \mathfrak{m}^{q}=J \mathfrak{m}^{q-1}$ since $A$ is homogeneous. Hence $A$ is $q$-linear by [4, Corollary 1.15].

Suppose that $c=n-d \geq 2$. The Alexander dual of $\Delta$ is defined by

$$
\Delta^{*}:=\{F \subseteq V: V \backslash F \notin \Delta\} .
$$

Then $\Delta^{*}$ is a simplicial complex on $V$ with $\operatorname{dim} k\left[\Delta^{*}\right]=c+d-q$, indeg $k\left[\Delta^{*}\right]=c$ and $\operatorname{codim} k\left[\Delta^{*}\right]=q$. Also, the Betti numbers of $k\left[\Delta^{*}\right]$ are given by the formula

$$
\beta_{i, j}\left(k\left[\Delta^{*}\right]\right)=\sum_{\substack{F \in \Delta \\ \#(F)=c+d-j}} \operatorname{dim}_{k} \widetilde{H}_{i-2}\left(\operatorname{link}_{\Delta} F ; k\right),
$$

where $\operatorname{link}_{\Delta}(F)=\{G \in \Delta: F \cap G=\emptyset, F \cup G \in \Delta\}$. Eagon and Reiner [3] proved that $k[\Delta]$ has linear resolution if and only if $k\left[\Delta^{*}\right]$ is Cohen-Macaulay; see [3, 16] for more details. Based upon this result, we can characterize the Alexander dual of Buchsbaum simplicial complexes with linear resolution as follows:

Theorem 1.4. Let $c, d, q$ be integers with $c \geq 2,2 \leq q \leq d$. Let $A=k[\Delta]$ be a $d$-dimensional Stanley-Reisner ring with $\operatorname{codim} A=c$ and indeg $A=q$, and let $\Delta^{*}$ denote the Alexander dual of $\Delta$. Put $A^{*}:=k\left[\Delta^{*}\right]$. Then the following conditions are equivalent:

(1) A is Buchsbaum with q-linear resolution.

(2) $A^{*}$ is Cohen-Macaulay with almost c-linear resolution, and the graded minimal free resolution of $A^{*}$ over $S$ can be written as follows:

$$
\begin{aligned}
0 \rightarrow F_{q} \rightarrow F_{q-1} & =S(-(c+q-2))^{\beta_{q-1}^{*}} \rightarrow \cdots \rightarrow F_{1}=S(-c)^{\beta_{1}^{*}} \rightarrow S \rightarrow A^{*} \rightarrow 0, \\
& \text { where } F_{q}=S(-(c+d))^{\beta^{*}} \oplus S(-(c+q-1))^{\beta^{*^{\prime}}} .
\end{aligned}
$$

When this is the case, $\beta^{*}=\operatorname{dim}_{k} H_{\mathfrak{m}}^{q-1}(A)$ and $\beta^{*^{\prime}}=\operatorname{dim}_{k} H_{\mathfrak{m}}^{d}(A)_{q-d-1}$.

Proof. We may assume that $A$ is $q$-linear and $A^{*}$ is Cohen-Macaulay. Note that $k[\Delta]$ is Buchsbaum if and only if $\Delta$ is pure and $\widetilde{H}_{i}\left(\operatorname{link}_{\Delta}(F) ; k\right)=0$ for every $F(\neq \emptyset) \in \Delta$ and for every $i<\operatorname{dim}_{\operatorname{link}}(F)$.

$(1) \Longrightarrow(2)$ : To see $(2)$, we must show that $\beta_{i j}\left(k\left[\Delta^{*}\right]\right)=0$ for all pairs $(i, j)$ with $0 \leq i \leq q$ and $j>c+i-1$ except $(i, j)=(q, c+d)$. 
Let $F$ be a face of $\Delta$ with $\#(F)=c+d-j$. First suppose that $j=c+d$. Then $F=\emptyset$. If $i \leq q-1$, then $\widetilde{H}_{i-2}(\Delta ; k)=0$ by Proposition 1.1. Next suppose that $c+i-1<j<c+d$. Then $F \neq \emptyset$. Since $i-2<j-c-1=d-\#(F)-1=\operatorname{dim}_{\operatorname{link}_{\Delta} F}$, the Buchsbaumness of $\Delta$ implies that $\widetilde{H}_{i-2}\left(\operatorname{link}_{\Delta} F ; k\right)=0$. Thus we get the required vanishing.

$(2) \Longrightarrow(1)$ : First, note that $\Delta$ is pure. Indeed, $I_{\Delta^{*}}$ is minimally generated by the elements $X_{j_{1}} \cdots X_{j_{c}}$ for which $V \backslash\left\{x_{j_{1}}, \ldots, x_{j_{c}}\right\}$ is a facet of $\Delta$.

To see the Buchsbaumness of $k[\Delta]$, let $F$ be any non-empty face of $\Delta$ and let $i$ be an integer with $i<d-\#(F)+1$. Put $j=c+d-\#(F)$. Then one can easily see that $\beta_{i j}\left(k\left[\Delta^{*}\right]\right)=0$ by the assumption $(2)$. This implies that $\widetilde{H}_{i-2}\left(\operatorname{link}_{\Delta}(F) ; k\right)=0$. Hence $k[\Delta]$ is Buchsbaum, as required. Putting $i=q, j=n(=c+d)$, we have

$$
\beta^{*}=\beta_{q, n}\left(k\left[\Delta^{*}\right]\right)=\operatorname{dim}_{k} \widetilde{H}_{q-2}(\Delta ; k)=\operatorname{dim}_{k} H_{\mathfrak{m}}^{q-1}(A) .
$$

On the other hand, since $a(k[\Delta]) \leq q-d-1$, we have $\widetilde{H}_{d-\#(F)-2}\left(\operatorname{link}_{\Delta} F ; k\right)=0$ for every face $F$ with $1 \leq \#(F) \leq d-q$. Thus we have

$$
\operatorname{dim}_{k}\left[H_{\mathfrak{m}}^{d}(k[\Delta])\right]_{q-d-1}=\sum_{F \in \Delta, \#(F)=d-q+1} \operatorname{dim}_{k} \widetilde{H}_{q-2}\left(\operatorname{link}_{\Delta} F ; k\right)=\beta^{*^{\prime}}
$$

by Hochster's formula on the Betti numbers.

\section{Buchsbaum Stanley-Reisner RINGs With Minimal MUltiplicity}

Let $A=k\left[A_{1}\right]$ be a homogeneous $k$-algebra of dimension $d$ with the unique homogeneous maximal ideal $\mathfrak{m}=A_{+}$. In [8], Goto proved an inequality

$$
e(A) \geq 1+\sum_{i=1}^{d-1}\left(\begin{array}{c}
d-1 \\
i-1
\end{array}\right) l_{A}\left(H_{\mathfrak{m}}^{i}(A)\right)
$$

and called the ring $A$ a Buchsbaum ring with minimal multiplicity if equality holds. Also, he proved that such an algebra has 2-linear resolution; see [7, 8].

In this section, in the class of Stanley-Reisner rings, we introduce the notion of Buchsbaum ring with minimal multiplicity of initial degree $q$ and prove that such a ring has $q$-linear resolution. Furthermore, we give several characterizations of this notion. In the following, let $c, d, q$ be integers with $c \geq 2,2 \leq q \leq d$. Also, let $k[\Delta]$ be a $d$-dimensional Buchsbaum Stanley-Reisner ring of a simplicial complex $\Delta$ on $V=\left\{x_{1}, \ldots, x_{n}\right\}$, and put $\mathfrak{m}=\left(X_{1}, \ldots, X_{n}\right) k[\Delta]$, the unique homogeneous maximal ideal of $k[\Delta]$. Put $\Gamma_{i}:=\operatorname{link}_{\Delta}\left(\left\{x_{i}\right\}\right)$ for each $i$, and let $\mathfrak{m}_{i}$ be the homogeneous maximal ideal of $k\left[\Gamma_{i}\right]$ for all $i$.

Let $A=k[\Delta]$ be as above with $\operatorname{codim} A=c$ and indeg $A=q$. Since $A$ is Buchsbaum, $k\left[\Gamma_{i}\right]$ is a $(d-1)$-dimensional Cohen-Macaulay ring with $\operatorname{codim} k\left[\Gamma_{i}\right]=$ $c$ and indeg $k\left[\Gamma_{i}\right] \geq q-1$. By [4, Corollary 1.11], we get

$$
e\left(k\left[\Gamma_{i}\right]\right) \geq\left(\begin{array}{c}
c+(q-1)-1 \\
(q-1)-1
\end{array}\right)=\left(\begin{array}{c}
c+q-2 \\
q-2
\end{array}\right) .
$$

On the other hand, counting the number of facets of $\Delta$, we have

$$
d \cdot e(A)=\sum_{i=1}^{n} e\left(k\left[\Gamma_{i}\right]\right) \geq(c+d)\left(\begin{array}{c}
c+q-2 \\
q-2
\end{array}\right) .
$$

From this, we can define the notion of minimal multiplicity of initial degree $q$. 
Proposition 2.1. Suppose that $A=k[\Delta]$ is Buchsbaum with $d=\operatorname{dim} A, c=$ $\operatorname{codim} A$ and $q=\operatorname{indeg} A$. Then

$$
e(A) \geq \frac{c+d}{d}\left(\begin{array}{c}
c+q-2 \\
q-2
\end{array}\right) .
$$

We say that $A$ has minimal multiplicity of initial degree $q$ if equality holds in Eq. (2.1). This notion for $q=2$ is equivalent to the notion of minimal multiplicity in the sense of Goto; see Example 3.1.

In the following, let us prove that any Buchsbaum Stanley-Reisner ring with minimal multiplicity of initial degree $q$ has $q$-linear resolution and characterize such rings. In order to do that, we need the following lemma.

Lemma 2.2 (cf. [10, Theorem (1.6)]). Suppose that $A=k[\Delta]$ is a d-dimensional Buchsbaum ring with $2 \leq q \leq d$. If $a\left(k\left[\Gamma_{i}\right]\right)=q-d-1$ for all $i$, then $A$ has $q$-linear resolution and $a(A)=q-d-2$.

Proof. By Hochster's formula, we have

$$
\begin{aligned}
F\left(H_{\mathfrak{m}}^{d}(A), t\right) & =\sum_{F \in \Delta} \operatorname{dim}_{k} \widetilde{H}_{d-\#(F)-1}\left(\operatorname{link}_{\Delta} F ; k\right)\left(\frac{t^{-1}}{1-t^{-1}}\right)^{\#(F)} ; \\
F\left(H_{\mathfrak{m}_{i}}^{d-1}\left(k\left[\Gamma_{i}\right]\right), t\right) & =\sum_{G \in \Gamma_{i}} \operatorname{dim}_{k} \widetilde{H}_{d-\#(G)-2}\left(\operatorname{link}_{\Gamma_{i}} G ; k\right)\left(\frac{t^{-1}}{1-t^{-1}}\right)^{\#(G)} .
\end{aligned}
$$

Claim 1: $\left[H_{\mathfrak{m}}^{d}(A)\right]_{j}=0$ for all $j=-1, \ldots, q-d-1$.

Now let $F$ be a face of $\Delta$ with $1 \leq \#(F) \leq d-q+1$. As $F$ contains a vertex of $\Delta$ (say $x_{i}$ ), if we put $G=F \backslash\left\{x_{i}\right\}$, then $G \in \Gamma_{i}$ and $\operatorname{link}_{\Gamma_{i}} G=\operatorname{link}_{\Delta} F$. If $G \neq \emptyset$, then $1 \leq \#(G)=\#(F)-1 \leq d-q$. Then

$$
\widetilde{H}_{d-\#(F)-1}\left(\operatorname{link}_{\Delta} F ; k\right)=\widetilde{H}_{d-\#(G)-2}\left(\operatorname{link}_{\Gamma_{i}} G ; k\right)=0
$$

because $\left[H_{\mathfrak{m}_{i}}^{d-1}\left(k\left[\Gamma_{i}\right]\right)\right]_{q-d}=0$. If $G=\emptyset$, then $F=\left\{x_{i}\right\}$. Thus

$$
\widetilde{H}_{d-\#(F)-1}\left(\operatorname{link}_{\Delta} F ; k\right)=\widetilde{H}_{d-2}\left(\Gamma_{i} ; k\right)=\left[H_{\mathfrak{m}_{i}}^{d-1}\left(k\left[\Gamma_{i}\right]\right)\right]_{0}=0 .
$$

Hence $\left[H_{\mathfrak{m}}^{d}(A)\right]_{j}=0$ by Hochster's formula.

Claim 2: $\left[H_{\mathfrak{m}}^{d}(A)\right]_{0} \cong \widetilde{H}_{d-1}(\Delta ; k)=0$.

Let $K_{A}$ be the graded canonical module of $A$. Then, by Claim $1,\left[K_{A}\right]_{1}=$ $\operatorname{Hom}_{k}\left(\left[H_{\mathfrak{m}}^{d}(A)\right]_{-1}, k\right)=0$. Since depth $K_{A}>0,\left[K_{A}\right]_{0} \subseteq \operatorname{Hom}_{A}\left(A / \mathfrak{m}, K_{A}\right)=0$, and thus $\left[H_{\mathfrak{m}}^{d}(A)\right]_{0}=0$.

By the above two claims, $a(A) \leq q-d-2$. By [11, Corollary 2.8], we have $\operatorname{reg} A \leq q-1$. Therefore $A$ is $q$-linear and $a(A)=q-d-2$, as required.

The following theorem is a main theorem in this paper.

Theorem 2.3. Suppose that $A=k[\Delta]$ is Buchsbaum with $\operatorname{dim} A=d, \operatorname{codim} A=c$ and $\operatorname{indeg} A=q$. Let $\Delta^{*}$ denote the Alexander dual of $\Delta$ and put

$$
h_{c, d, q}=\frac{(c+q-2) \cdots(c+1) c}{d(d-1) \cdots(d-q+2)} .
$$

Then the following conditions are equivalent:

(1) A has minimal multiplicity of initial degree $q$.

(2) $A$ has q-linear resolution and $\operatorname{dim}_{k} \widetilde{H}_{q-2}(\Delta ; k)=h_{c, d, q}$. 
(3) The h-vector of $A$ is

$$
\left(1, c, \ldots,\left(\begin{array}{c}
c+q-2 \\
q-1
\end{array}\right),-\left(\begin{array}{l}
d \\
q
\end{array}\right) h,\left(\begin{array}{c}
d \\
q+1
\end{array}\right) h, \ldots,(-1)^{d-q+1}\left(\begin{array}{l}
d \\
d
\end{array}\right) h\right),
$$

where $h=h_{c, d, q}$.

(4) $k\left[\operatorname{link}_{\Delta}\left\{x_{i}\right\}\right]$ has $(q-1)$-linear resolution for all $i$.

(5) $a\left(k\left[\operatorname{link}_{\Delta}\left\{x_{i}\right\}\right]\right)=q-d-1$ for all $i$.

(6) $a(A)=q-d-2$.

(7) $k\left[\Delta^{*}\right]$ is Cohen-Macaulay with pure and almost linear resolution and with $a\left(k\left[\Delta^{*}\right]\right)=0$, that is, the graded minimal free resolution of $k\left[\Delta^{*}\right]$ can be written as follows:

$$
0 \rightarrow S(-(c+d))^{\beta_{q}^{*}} \rightarrow S(-(c+q-2))^{\beta_{q-1}^{*}} \rightarrow \cdots \rightarrow S(-c)^{\beta_{1}^{*}} \rightarrow S \rightarrow k\left[\Delta^{*}\right] \rightarrow 0 .
$$

When this is the case, $\beta_{q}^{*}=h_{c, d, q}$.

Proof. Suppose (1). Then $e\left(k\left[\Gamma_{i}\right]\right)=\left(\begin{array}{c}c+q-2 \\ q-2\end{array}\right)$ for all $i$. This implies that $k\left[\Gamma_{i}\right]$ is $(q-1)$-linear. Also, this condition is equivalent to $a\left(k\left[\Gamma_{i}\right]\right)=q-d-1$, since $k\left[\Gamma_{i}\right]$ is Cohen-Macaulay. Thus we get $(1) \Rightarrow(4) \Leftrightarrow(5) \Rightarrow(6)$ by Lemma 2.2 , Similarly, one can prove $(6) \Rightarrow(5)$.

We next show that (1) $\Rightarrow(2) \Rightarrow(3) \Rightarrow(1)$. If we suppose (1), by the above argument, $A$ is $q$-linear. Putting $h=\operatorname{dim}_{k} \widetilde{H}_{q-2}(\Delta ; k)$, we obtain that

$$
\frac{c+d}{d}\left(\begin{array}{c}
c+q-2 \\
q-2
\end{array}\right)=e(A)=\left(\begin{array}{c}
c+q-1 \\
q-1
\end{array}\right)-h\left(\begin{array}{l}
d-1 \\
q-1
\end{array}\right)
$$

by Theorem 1.3. This implies that $h=h_{c, d, q}$. In particular, we get (2). Also, $(2) \Rightarrow(3)$ follows from Theorem 1.3 If we suppose (3), then we have

$$
e(A)=\sum_{i=0}^{d} h_{i}=\left(\begin{array}{c}
c+q-1 \\
q-1
\end{array}\right)-\left(\begin{array}{l}
d-1 \\
q-1
\end{array}\right) h_{c, d, q}=\frac{c+d}{d}\left(\begin{array}{c}
c+q-2 \\
q-2
\end{array}\right) .
$$

Hence we get (1).

To complete the proof, we must show that $(5),(6) \Rightarrow(7) \Rightarrow(2)$. Suppose that (5) and (6) are satisfied. By Lemma 2.2, $A$ is $q$-linear. Thus by Theorem 1.4. the Alexander dual $k\left[\Delta^{*}\right]$ is Cohen-Macaulay with almost linear resolution. Furthermore, since $\beta^{*^{\prime}}=\operatorname{dim}_{k} H_{\mathfrak{m}}^{d}(A)_{q-d-1}=0$ by (6), the graded minimal free resolution of $k\left[\Delta^{*}\right]$ is the required form. Hence we get (7). Conversely, suppose (7). By Theorem [1.4, $A=k[\Delta]$ is $q$-linear. On the other hand, since $k\left[\Delta^{*}\right]$ has pure resolution of type $\left(c_{1}, \ldots, c_{q}\right)=(c, c+1, \ldots, c+q-2, c+d)$, by Herzog-Kühl [9], we have

$$
\beta_{q}^{*}=(-1)^{q+1} \prod_{j=1}^{q-1} \frac{c_{j}}{c_{j}-c_{q}}=h_{c, d, q} .
$$

Combining with $\operatorname{dim}_{k} \widetilde{H}_{q-2}(\Delta ; k)=\beta_{q}^{*}$, we obtain the required assertion.

\section{EXAMPLES}

In the following, we give several examples of Buchsbaum Stanley-Reisner rings with minimal multiplicity. Put $[n]=\{1,2, \ldots, n\}$ for any positive integer $n$. We say that a simplicial complex $\Delta$ is spanned by a set $S$ if $S$ is the set of facets of $\Delta$. 
Example 3.1. Let $A=k[\Delta]$ be a $d$-dimensional Buchsbaum Stanley-Reisner ring with indeg $A \geq 2$. Then the following conditions are equivalent:

(1) $A$ has minimal multiplicity in the sense of Goto 8 .

(2) $A$ has minimal multiplicity of initial degree 2.

(3) $\Delta$ is a finite disjoint union of $(d-1)$-simplexes.

When this is the case, $\#\{$ the connected components of $\Delta\}=e(A)$.

Proof. $(2) \Longrightarrow(3)$ : Since each facet of $\Delta$ is a $(d-1)$-simplex, we have $n \leq d e$ by counting the vertices of $\Delta$. Equality holds if and only if $\Delta$ is a disjoint union of all facets. (3) $\Longrightarrow(1)$ is easy. To see $(1) \Longrightarrow(2)$, suppose that $A$ has minimal multiplicity. Then it is well known that it has 2-linear resolution and $e:=e(A)=$ $1+h$ and $I(A)=(d-1) h$, where $h=\operatorname{dim}_{k} H_{\mathfrak{m}}^{1}(A)$. Thus $n=\operatorname{dim}_{k} A_{1}=e+d-$ $1+I(A)=d e$, as required.

Example 3.2 ([15, Theorem 3.3]). Let $n$ be an integer such that $n>3$, and suppose that $2 n+1$ is a prime number. Let $\Delta$ be the simplicial complex on $V=[n]$ which is spanned by

$$
\begin{aligned}
S= & \{\{a, b, a+b\}: 1 \leq a<b, a+b \leq n\} \\
& \cup\{\{a, b, c\}: 1 \leq a<b<c \leq n, a+b+c=2 n+1\} .
\end{aligned}
$$

Then $A=k[\Delta]$ is a 3 -dimensional Buchsbaum Stanley-Reisner ring with minimal multiplicity of initial degree 3 .

Proof. By [15], $A$ is Buchsbaum with $e(A)=\frac{n(n-2)}{3}=\frac{c+d}{d}\left(\begin{array}{c}c+q-2 \\ q-2\end{array}\right)$ and initial degree 3 . Thus we get the assertion by definition.

Example 3.3 (Hibi, 10]). Let $d \geq 2$ be an integer, and let $k$ be a field. Put $n=2 d-1$ and $V=\{1,2, \ldots, n\}$. Let $\Delta$ be the simplicial complex which is spanned by $S=\{\{\bar{i}, \overline{i+1}, \ldots, \overline{i+d-1}\}: i=1,2, \ldots, 2 d-1\}$, where $\bar{p}$ stands for $q \in V$ with $p \equiv q(\bmod 2 d-1)$. Then $A=k[\Delta]$ is a $d$-dimensional Buchsbaum Stanley-Reisner ring with minimal multiplicity of initial degree 3.

Proof. First note that $A$ is equidimensional with $c=n-d=d-1$ and indeg $A=3$ because $\{1,2, d\} \notin \Delta$. Also, since

$$
\operatorname{link}_{\Delta}\{1\}=\{2, \ldots, d\} \cup\{2 d-1,2, \ldots, d-1\} \cup \cdots \cup\{d+1, d+2, \ldots, 2 d-1\},
$$

$k\left[\operatorname{link}_{\Delta}\{1\}\right]$ is Cohen-Macaulay with 2-linear resolution and so is $k\left[\operatorname{link}_{\Delta}\{i\}\right]$; see [6, 17. Thus the assertion follows from Theorem 2.3(4).

Hibi posed the following problem in [10].

Problem 3.4 (Hibi). Construct a non-Cohen-Macaulay Buchsbaum complex of dimension $d-1$ with $q$-linear resolution for any given integers $q$, $d$ with $2 \leq q \leq d$.

The next example gives a complete answer to the above problem.

Example 3.5 (The Alexander dual of a cyclic polytope). Let $q, d$ be given integers with $2 \leq q \leq d$. Put $n=2 d-q+2$ and $f=2(d-q+1)$. Let $\Delta$ be the Alexander dual of the boundary complex $\Gamma$ of a cyclic polytope $C(n, f)$ with $n$ vertices. Then $k[\Delta]$ is a $d$-dimensional Buchsbaum Stanley-Reisner ring with minimal multiplicity of initial degree $q$. 
Proof. It is known that $k[\Gamma]$ is an $f$-dimensional Gorenstein Stanley-Reisner ring with $a(k[\Gamma])=0$. Also, we have indeg $k[\Gamma]=f / 2+1=n-d$ since $h_{i}(\Gamma)=$ $\left(\begin{array}{c}n-f+i-1 \\ i\end{array}\right)$ for all $i$. This implies that $k[\Gamma]$ has almost $(n-d)$-linear resolution (see $[12,18]$. . On the other hand, since $\operatorname{dim} k[\Delta]=d$ and indeg $k[\Delta]=q$, the assertion follows from Theorem $2.3(7)$.

The next example gives Buchsbaum Stanley-Reisner rings with minimal multiplicity of higher initial degree.

Example 3.6 (Bruns-Hibi, [2, Proposition 3.2]). Let $n \geq 6$ be an even integer, and let $k$ be a field. Let $\Gamma$ be the simplicial complex which is spanned by

$$
\{\bar{i}, \overline{i+1}, \overline{i+2}\},\{\bar{i}, \overline{i+1}, \overline{i+4}\}, \ldots,\{\bar{i}, \overline{i+1}, \overline{i+(n-2)}\}, \quad i=1, \ldots, n,
$$

where $\bar{p}$ stands for $q \in[n]$ with $p \equiv q(\bmod n)$. Also, let $\Delta$ be the Alexander dual of $\Gamma$. Then $A=k[\Delta]$ is an $(n-3)$-dimensional Buchsbaum Stanley-Reisner ring with minimal multiplicity of initial degree $(n-3)$. Also, $\operatorname{codim} A=3$ and $\operatorname{dim}_{k} A_{1}=n$.

Proof. The required assertion follows from Theorem 2.3(7).

\section{REFERENCES}

[1] W. Bruns and J. Herzog, Cohen-Macaulay Rings, Cambridge University Press, Cambridge, New York, Sydney, 1993. MR1251956 (95h:13020)

[2] W. Bruns and T. Hibi, Stanley-Reisner rings with pure resolutions, Communications in Algebra 23-4 (1995), 1201-1217. MR1317395 (96h:13059)

[3] J. A. Eagon and V. Reiner, Resolutions of Stanley-Reisner rings and Alexander duality, J. Pure and Applied Algebra 130 (1998), 265-275. MR.1633767 (99h:13017)

[4] D. Eisenbud and S. Goto, Linear free resolutions and minimal multiplicity, J. Algebra 88 (1984), 89-133. MR0741934 (85f:13023)

[5] R. Fröberg, Rings with monomial relations having linear resolutions, J. Pure Appl. Algebra 38 (1985), 235-241. MR0814179 (87b:13020)

[6] R. Fröberg, On Stanley-Reisner rings, in "Topics in algebra," Banach Center Publications, No. 26, PWN-Polish Scientific Publishers, Warsaw, 1990, pp. 57-70. MR1171260 (93f:13009)

[7] S. Goto, Buchsbaum rings of maximal embedding dimension, J. Algebra 76 (1982), 383-399. MR0661862 (83k:13014)

[8] S. Goto, On the associated graded rings of parameter ideals in Buchsbaum rings, J. Algebra 85 (1983), 490-534. MR0725097 (85d:13032)

[9] J. Herzog and M. Kühl, On the Betti numbers of finite pure and linear resolutions, Comm. Alg. 22 (1984), 1627-1646. MR0743307 (85e:13021)

[10] T. Hibi, Buchsbaum complexes with linear resolutions, J. Algebra 179 (1996), 127-136. MR.1367844 (97f:13021)

[11] L. T. Hoa and C. Miyazaki, Bounds on Castelnuovo-Mumford regularity for generalized Cohen-Macaulay graded rings, Math. Ann. 301 (1995), 587-598. MR.1324528 (96e:13019)

[12] P. Schenzel, Über die freien Auflösungen extremaler Cohen-Macauley-Ringe, J. Algebra 64 (1980), 93-101. MR0575785 (81j:13024)

[13] R. P. Stanley, Combinatorics and Commutative Algebra, Second Edition, Birkhauser, Boston/Basel/Stuttgart, 1996. MR1453579 (98h:05001)

[14] J. Stückrad and W. Vogel, Buchsbaum Rings and Applications, Springer-Verlag, Berlin, Heidelberg, New York, 1986. MR0881220 (88h:13011a)

[15] N. Terai, On h-vectors of Buchsbaum Stanley-Reisner rings, Hokkaido Math. J. 25 (1996), 137-148. MR1376497 (97b:13028)

[16] N. Terai, Alexander duality theorem and Stanley-Reisner ring, in Free resolutions of coordinate rings of projective varieties and related topics (Japanese) (Kyoto, 1998), R.I.M.S. No. 1078 (1999), pp. 174-184. MR.1715588(2001f:13033) 
[17] N. Terai, Eisenbud-Goto inequality for Stanley-Reisner rings, Geometric and combinatorial aspects of commutative algebra (Messina, 1999), 379-391, Lecture Notes in Pure and Appl. Math., 217, Dekker, New York, 2001. MR1824243 (2002a:13021)

[18] N. Terai and T. Hibi, Computation of Betti numbers of monomial ideals associated with cyclic polytopes, Discrete and Computational Geometry 15 (1996), 287-295. MR.1380395 (96k:13021)

Department of Mathematics, Faculty of Culture and Education, Saga University, SAGA $840-8502$, JAPAN

E-mail address: terai@cc.saga-u.ac.jp

Graduate School of Mathematics, Nagoya University, Nagoya 464-8602, Japan

E-mail address: yoshida@math.nagoya-u.ac.jp 